no salpingitis, no threatenings of phlegmasia alba dolens? Is it not possible that with a more rigid system of sterilizing our instruments, our hands, and our midwifery bag, we might reduce that troublesome morbidity also to a vanishing point? Credo.

1 Lancet, 1867 , i, p. 326 ; ii. p. 353.2 Trans. Edin. Obst. Soc., vi, p. 3, r88 3 Ibid., xv, p. 119. 1890. \& Centralbl. f. Gynäk, xvi, p. 440, 1892. 5 Ibid., xix, p. 1033, 1895. 6 Ibid., p. 1062. 7 Ibid., p. 1361 . 8 Ibid., XXvil, p. 1240, 1903.

\section{A (x)erture}

\author{
ON
}

\section{THE VALUE OF THE IMPERFECTLY-DESCENDED TESTIS, THE ADVISABILITY OF OPERATION, AND THE VALUE OF THE OPERATIONS PERFORMED FOR ITS RELIEF.}

A Post.graduate Lecture delivered at the Hospital for Sick Children, Great Ormond Street.

Bx EDRED M. CORNER, B.Sc.Lond., M.A., M.B., B.C. (CaNTaB.), F.R.C.S.ENG

Assistant Surgeon, Hospital for Sick Chisdren, Great Ormond Street surgeou to Out-patients, St Thomas Hospital; Erasmus Wilson Lecturer, Royal College of Surgeons of England.

Gentlemen, - It is my intention to put before you as clear an account as is pussible of the physiological value of the imperfectly-descended testicle, both before and after operation. The more common name of "undescended testicle" has been discarded as clumsy and inexact, and the above one has been employed in its stead. The lecture naturally falls into the three parts indicated by the title, the intermediate section dealing with the advisibility of operation so leading from the one to the other of the above divisions. The first section on the value of the imperfectly-descended testis cannot be anything lise adequately treated in the scope of this lecture, and an attempt only has been made to place before you some of the grounds which lead to the belief that the imperfect descent of the testicle is only the outward, anatomical and visible sign of an inward and much more extensive physiological defect. In order to estimate the value of the testis it is necessary to make inquiries along two lines: first, the capacity of the gland to produce spermatozoa, and, secondly, its effect on the perfection of the so-called secondary sexual characters of the organism, of which two the Jatter is by a very long way the more important with regard to the imperfectly-descended testis, and will naturally be considered first.

The Value of the Imperfectly-descended Testis.

It has been the custom of late to regard the testes and ovaries as responsible for the differences that exist between the sexes and to overlook the more obscure and probably more far-reaching cause or causes which have led to the differentiation of those glands themselves, and which may have given rise also to other sexually distinctive formations. On the latter view the testes or ovaries become merely incidents, though by far the most important, in the sexual development of the individual.

To bring before you the great imfortance of this more widely-acting and general factor in the sexual development of the individual or ontogeny, examples of very early sexual differentiation will be brought to your notice. The endothelium from which these glands are developed has been called the "germinal" epithelium, and is situated along the inner side of the Wolfian body or primitive kidney. The cells first elongate, becoming columnar; subsequently by division two layers are formed instead of one, Jurther multiplication results in the presence of several rows of cells. The underlying tissue or mesoblast also becomes thickened and helps to give prominence to the germinal endothelium, so forming the genital ridge. At this very early stage of fetal life and before the generative glands or gonads are themselves formed, the first known sexual distinction can be found. In the female sex the germinal epithelium is several layers thick and grows down into the subjacent tissue or mesoblastic stroma in the form of columns of cells. In the male, the germinal epithelium is not nearly so thick and only small glands of cells grow down into the stroma. It will be observed that the distinction lies rather in quantity than in quality. The same property that gave rise to the testes and ovaries also causes the formation of the other external and internal genitalia, so that it is very do'abtful if the pr: noipal glands have anything to do with influencing these formations, which apparently arise independently. A good deal later, both glands undergo considerable changes in position. The ovary descends only as far as the side of the uterus, very occasionally it reaches as far as the groin. The testis, during the sixth month of fetal life, is at the internal abdominal ring, during the seventh month it is in the inguinal canal, and in the eighth month it enters the scrotum. These facts clearly indicate that the cause of the primary sexual characters of the organism are the results of inherent tendencies of the ovum, and are not due to any action of the ovaries or testes. Professor Arthur ' $i$ 'homson, of Oxford, emphasizes this early differentiation of sex by showing that sexual distinctions can be found in the pelves at an early date when presumably the generative glands or gonads are as set inactive. "My results, obtained independently, strikingly confirm what I believe is Fehling's main contention, that the differences of form and appearance are such as enable the observer to discriminate between the pelves of the male and the female as early as the third month" of fetal li'e.

Nowadays a gland is looked upon as having both an internal as well as an external secretion. And it has been supposed that the secondary sexual characters are developed as the result of the action of the former. As yet we know nothing of the possible action of internal secretions during intrauterine life. But as in that period we are supposed to climb up our anatomical genealogical tree, so may we also ascend a similar physiological staircase. But, at least, it is unlikely that a gland can accomplish much before it is fully differentiated and functionally active. It may be further pointed out that some of the so-called secondary sexual differences exist at birth. For instance, male infants are already heavier and taller than female, their chest girth is greater; later they show more variations, different rates of growth, different ages for the incidence of puberty, etc. It may in consequence be suggested that many, if not all, of these so-called secondary sexual distinctions are independent for their origin of the testes and ovaries. Rather do they give the idea ot being dependant on some innate property of the ovum, being found early and probably before those glands are a'ctive. At puberty all sexual differences are exaggerated and some new ones initiated, such as the growth of hair on the face, the breaking of the voice, etc. If the testes are removed before puberty many of the male characters do not appear to develop at all. But if a carfful examination is made it will be found that these features are not absent, but are only very slightly developed. For example, one may instance the outward bodily form of the eunuchs of the East, or the more familiar bullock. But we are not interested at this moment in cases of absence of the testes, but rather in poorly-developed ones. For their origin the secondary sexual features are most probably dependent on the same obscure cause that brings about the advent of puberty, as well as the changes in the testes themselves; but, for their perfectation the good development of those generative glands is necessary. When, however, these secondary characters are once established they seem to be independent of the integrity of those same glands. Now, by far the most important point in the treatment of imperfectlydescended testes is how to make the best of this factor for the development of manly characters, or how to estimate the value of the internal secretion of the testes. That an imperfectly-descended testis can so bring about the acquisition of manly beauty is certain. For instance, in its widest sense, one sees many and widely-varying degrees in the sexual development of cryptorchids. But this may be more neatly illustrated in animals than in man. A horse when young had had its only descended testis removed. The animal soon displayed the so-called rig or vicious and troublesome tendencies. Mr. Hobday, the veterinary surgeon, explored the inguinal canal and removed a mass at the top, which he thought might the missing testis. For two years the animal remained fairly tractable, but then recommenced his rig habits. Mr. Hobday opened the abdomen and successfully removed the hidden testis. After this the animal became quiet and was no longer subject to attacks of sexual excitement.

The above must suffice for the subject of the internal secretion of the testicle and serves well enough to indicate that both primary and secondary sexual characters probably originate independently of the growth of the testes, though for their perfect development that growth is necessary. Again, the value of an imperfectly-descended testis depends almost entirely on this internal secretion and it must be our foremost duty to do our best to foster it.

The most important function of the normal testis is that of producing spermatozina and so ensuring the perretuation of 
the race. In this respect an imperfectly-descended testis is practically lacking. Few, if any, absolutely authentic cases of an imperfectly-descended testis producing spermatozoa have been recorded. But a few instances of cryptorchids becoming parents have been reported. These and some like cases go to show that although the imperfectly-descended testis is sufficiently highly developed to subserve this function in rare instances, one can never deny the possibility of the rare occurrence of such an event. The evidence in favour of the existence of such a period of prosperity or activity goes to prove also that it is only of very short duration as the organ speedily undergoes retrograde changes.

I would therefore look upon the descent of the testis as the neressary accompaniment of the proper or full development of that organ. And that its imperfect descent is like a cleft of the palate in the development of the upper jaw, or the dislocation of the hip in the evolution of the thigh, or talipes equino-varus in that of the foot. The simile is added to by the fact that $\mathrm{n}$ y of these errors in ontogeny may be unilateral or bilateral. Although the testis is sometimes atrophic or imperfectly developed when fully descended, perfect development never goes with imperfect descent. There seems to be mo obvious reason for this; and the fact still further strengthens the idea that in these cases an anatomical and a physiological malformation go together.

To sum up, the imperiectly-descended testis can very rarely or practically never produces spermatozoa, though there is a faint possibility of this occurring about the age of twenty and lasting a year or two. But it is endowed with very various capabilitit $s$ with regard to assisting the development and perfectation of other sexual features. The extent of these powers it is impossible to estimate, as the failure of the testis may be due to the same fundamental cause that has led to the absence of beard, male voice, etc. When the condition is bilateral the outlook is bad, as there is no known remedy for errors of development, But, if the failure is only unilateral, there need be no alarm for the perpetuation of the species and the assumption of manly grace.

The Significance of Pain in the Imperfectly-descended Testis.

The symptom of pain in these cases is one on which I believe a good deal of reliance may be placed. The most obvious cause of pain is a direct injury to the organ, either from a blow or sudden and violent flexion of the thigh. Except that they may cause or hitis these are not very important or farreaching in their effects. Often-repeated slight injuries are much more important in leading to sclerosis of the organ. But when one considers the matter quite dispassionately, it is hard to conceive that the imperfectly-descended testis is really more liable, if indeed it is not less liable, to slight injuries than the gland that is normally situated. One is therefore inclined to give up this most popular notion of the cause of the sclerosis and atrophy of the imperfectly-descended testis. Now, it is perfectly well recognized that fibrosis is no congenital abnormality. How then does this condition come about and also so quietly? I believe that an explanation can be offered for this. At St. Thomas's Hospital, Great Ormond Street, and in practice I have done exactly 30 operations for this condition. And in no less than $16 \mathrm{I}$ have noted that the testis had undergone some torsion of the cord. More than this, it was noted that a distinct history of attacks of pain, frequently on exertion, was almost always accompanied with the discovery of the torsion at the operation ; and one cannot help correlating the two things and inferring that the pain was due to an increase of the twist. And the very anatomical causes that would bring about the torsion, namely, "movements and exertion," are the very ones that have been employed, and so are most likely to have increased the twist.

If one considers the course that far the majority of imperfectly-descended testes take at the groin, it will be seen that lying on the external oblique, they tend to ascend towards the anterior superior spine of the ilium on account of the movements of the flexion of the thigh, like a femoral hernia does. Such a course will lead to the formation of a more or less of a fixed point for the cord at the external abdominal ring, and the movements of the thigh will tend to roll the testis on the abdomen, so producing torsion, which a sudden violent exertion may increase sufficiently to beget a stound of pain. If one examines the recorded cases of torsion of testis, it is found that a number of the cases give a history of many previous attacks of pain. And finally the last attack brings about an acute strangulation. At the oper- ation, one finds perhaps as many as three or four complete twists of the cord. Yet one cannot suppose for a moment that the testis suddenly spun round giddily four times, but rather that these turns represent the total aggregate of many twists, spread over a long period of time. Imperfectlydescended testes seem very prone to suffer torsion, and the majority of cases of acute torsion of the testis have occurred in this variety. I would therefore like to put this hypothesis forward to explain the occurrence of attacks of pain in the testis, and also the frequency of fibrosis of the organ as a result of the twist interfering with the venous return from the imperfectly-descended gland.

The presence of the twist is usually easily noted if the tunica vaginalis is opened at the earliest opportunity at the operation and the position of the testis and epididymis noted. A little care is necessary to check any errors that a superficial examination sometimes leads one to make. On this hypothesis, pain takes a somewhat more serious appearance, as it indicates that there $i *$ in all probability a mechanism at work which will sooner or later bring about physiological destruc. tion of the already under-developed gland. Its presence, even in a mild form, should raise the question of speedy operation.

The significance of pain in these cases leads to the question as to whether an "undescended" testis should ever be left alone. And it seems, in the present state of our knowledge. that only harm is likely to accrue to the organ from its remaining in such a pnsition. Exception must be made of those instances in which the gland is nearly at the bottom of the scrotum. On the whole, it seems that the imperfectlydescended testis has a better chance of developing to the fullest extent of its limited capacity by being returned to the abdomen. And though direct proof is wanting, the sooner that this is done the better. From experimental knowledge it does not seem that it will even then progress to the extent of becoming spermatogenetic, this function seems, in almost all cases, quite beyond its capacity. As bas been already said, the most important power of the imperfectly-descended testis is that of ministering to the development of the secondary sexual characters, and beyond this no more can be expected. The whole question is, "Is this best obtained by protecting the organ from pathological change such as in the abdomen?" For my own part I think so. It is very difficult to obtain direct proof in support of this on account of the long time that must elapse before results show themselves. There is no evidence that the testis develops any further or any less far just outside the abdomen than it does just inside. And the latter position has the advantage of being, so far as we know, more or less out of the way of harm. There is certainly no evidence that the retained testis is more liable to undergo malignant change than the fully.descended and developed organ. Therefore, it seems best to return them to the abdomen.

There is also another and a very important factor in deciding on the performance of an operation. This is the presence or not of a hernia. Out of the 30 cases on which I have operated no less than 22 had communications between the tunica vaginalis and the peritoneal cavity. In some it was found that a hernia had previously come down. The presence of this communication is found the more frequently that it is looked for. The presence of an imperfectly-descended testis in almost every case contraindicates the use of a truss, if it does not do so in everv case on account of the injurious pressure that is exerted on the vessels of the spermatic cord.

It is almost needless to point out that some of the most serious cases of the strangulation of a hernia occur in conjunction with imperfect descent of the testis. The presence of a hernia is in consequence a most powerful incentive to operation. And as it occurs in so large a percentage, in reality or in potential, there really seems but little more needed to urge the need of operative interference. Beyond what has been said of the significance of the pain and the nearly universal presence of a hernial sac, potential or otherwise, no more will be added.

To sum up, if the testis is allowed to remain long in its imperfectly-descended position it will become largely functionless on account of undergrowing sclerosis, which may be hastened by torsion of the cord. Attacks of pain probably indicate spasmodic increments of that torsion, and abortive suicidal attempts at strangulation by ime organ. Also, a hernia sac is found in about 70 per cent. or so of the cases. Hence operation should be considered in every case of imperfectlydeseended testis and adopted $\vdots a$ a large number. 
Operations.

Before considering the various operations that have been performed for the relief of this condition, let me again recall to you the fact that we are dealing with an organ which can practically never become spermatogenetic, certainly for more than a year or two, but whose internal secretion may be of the greatest value to the organism.

\section{Orchidopexy or Orchidorrhaphy.}

By these terms are meant the fixation of the testis at the bottom of the scrotum. The chief reasons that have given rise to this practice are, first, the organ is placed in the natural anatomical position; and, secondly, in such a position it is hoped that it may develop properly and also cease to worry the patient. Unfortunately, after the operation, the testis often does not remain in the new position, but reascends; again, it never attains the size and development of the normal organ, but becomes smaller and harder from fibrosis probably secondary to the operative measures. Fortunately I have had the unique opportunity of examining microscopically a testis which had been successfully sutured in the scrotum two years previously. There was hardly a trace of testicular tissue left! It is therefore a great deal more than doubtful if a physiological triumph can be obtained by these means, that is, orchidopexy. Neither can an intelligent patient's mind be soothed by the presence of a small hard mass in one side of his scrotum, in striking contrast to the healthy testis on the other side. The presence of tension such as demands the employment of some rack or mechanical contrivance, to retain the organ in position is far more likely to do harm than good, and is a distinct contraindication to the operation.

The most favourable class of case that can be selected for this operation is that in which a fairly large hernia coexists with an imperfectly-descended testis and the tissues have been slowly stretched by the hernia. When the sac has been divided and freed, it is extraordinary with what ease the testis can be brought sometimes into the scrotum. In these cases the testis seems also to be the best developed. The next best class is that in which the testis has been driven far up the groin in the direction of the anterior superior spine, much the same as the femoral hernia is. Unfortunately in this position the testis is frequently sclerosed, although there is sometimes sufficient length of cord for an easy transplantation. And still more unfortunately, the mere size or consistence of the organ is no guide at all as to its functional capacities.

As the operation of orchidopexy necessitates considerable dissection for freeing the cord sufficiently to allow of the transplanting of the organ, with it is bound up the question of the reaction of the testis to interference with its blood supply and duct, the vas deferens.

$$
\text { A.-The Blood Supply. }
$$

In the operation for the relief of varicocele, the spermatic artery and the pampiniform plexus of veins are resected, whilst the vessels with the vas are left. During my tenure of the offices of Surgical Registrar and Resident Assistant Surgeon to St. Thomas's Hospital, my attention was attracted to the fact that a considerable number of those who had had a varicocele removed, in the course of a few months or a year or two had hardening and diminution in size of the testicle of that side. The frequency of such cases is infinitely greater than is suspected. A somewhat similar result was seen after radical cure of hernia in which the veins had been at all extensively removed. Halsted's operation is the one in which this was the most frequent. These instances show that he surgeon who aims at the improved nutrition of the testis, as in orchidopexy, must be extremely careful in dealing with its nutrient vessels. I may add that in one case an atrophic testis was excised along with a huge varicocele. Microscopic sections showed similar changes of fibrosis, absence of spermatozoa, and extreme scantiness of true testicular tissues.

\section{B.-Division of the Vas Deferens.}

It has been my misfortune on two occasions whilst performing the operation of radical cure of an inguinal hernia to divide the vas deferens. One of these cases I have seen four years later and there was no obvious change in the testicle. Similarly, nothing could be detected in the other case. Twoand-a-half years ago in doing a prostatectomy the vasa efferentia were torn across. There is no noticeable testicular change $n: T$ n r is there any diminution of sexual desire. The mirroscopical alterations that occur as a consequence in the gland are unknown. But I believe that fatty degeneration takes place which accounts for the absence of any obvious change in volume. Degeneration of the gland structures also explains the rarity of the formation of retention cysts.

Cases for orchidopexy must be very carefully selected with regard to all factors that might interfere with the fullest development which the gland is capable of. Regarding the frequent anatomical and almost invariable physiological failure of this operation, and the appearance of regression rather than further development of the testis, and that on the view put forward the gland is originally both anatomically and physiologically malformed, orchidopexy will be an operation which is but rarely called for.

Orchidectomy.

In the next series, consisting of 16 cases, the organ was excised. This was done for a variety of reasons, such as acute torsion of the cord, inability to transplant the gland into the scrotum, severe neuralgia, etc. In none of these, so far as I am aware, did the microscopic examination prove them to be spermatogenetic, even though many of the subjects were over puberty. The ages varied from $31^{7}$ to 46 . There was no evidence to think that wrong action had been taken, but looking back one cannot but help feeling that some would have been better if the gland had been put back in the abdomen. When the secondary sexual characters are well developed and after the age of 23 or so has been passed, that is apparently the only time when the organ has any chance of becoming spermatogenetic, the gland may be sacrificed on one side if a more radical cure can be done to a hernia or for a like reason. From the point of view of the individual the course of his imperfectly-descended testis has been run, and he will experience no harm bodily, and most probably mentally, from its removal.

\section{Orchidocoelioplasty.}

In my later series of operations for this condition the testis has been returned to the abdomen. Experimentally a testis returned to the abdomen does undergo some development, though not so much as does a normally situated gland. One returned to the abdomen after puberty soon loses its spermatogenetic capabilities, becoming microscopically like the imperfectly-descended organ. That an abdominal testis can produce sexual excitement is seen from the case of Mr. Hobday's horse already quoted. And double congenital cryptorchids have been known to develop manly characters, and have been alleged to have become parents. Therefore, it would appear that there is some benefit to the individual if the testicle be returned to the abdomen. And, as we are quite unable to accurately gauge the true value of an immature testis, it seems that we have no right to deny to the subject of that condition any value that may accrue to him from the presence of the gland. Naturally, many years must elapse before any definite results can be brought forward in support of this contention. And we have at present to work rather on a priori grounds. Apparently this operation has several advantages over the other two. The operation is easily and quickly performed, there need be no fear of interfering with the vascular supply of the gland or injury to its duct, the inguinal canal can be completely closed, the subject can receive all the benefit that the gland can give him, there seems to be very little opportunity offered for any steps of the operation to miscarry or fail, and so forth. Orchitis need not result from the procedure. And there is no evidence that the abdominal testis is more prone to undergo malignant change than the fully scrotal one. It, in consequence, seems advisable, whenever the testis cannot be quite easily brought into the scrotum and the subject is under 23, to return the organ to the abdomen.

The Value of the Abdominally-Replaced Testis. In this connexion it will be valuable from the point of view of prognosis that the value of the abdominally-placed testis, whether primarily or secondarily so, should be shortly discussed. Mr. Joseph Griffiths, as the result of experimental work, found that "when the testis of a young animal is replaced within the abdomen it undergoes but little change growing somewhat but not so much as the normally placed organ until the onset of puberty." "After the onset of puberty it continues to grow to some extent, though but little; the seminal tubules are seen to be lined by a single layer of columnar epithelium lying on the tunica propria with delicate prolongations extending into and occupying the lumen of the tubules; the central cells, from which in the normal state spermatozoa are produced, do not exist, and 
spermatozoa are accordingly not forthcoming." "The testicle remains in this state, at least we have no evidence that it undergoes further change." A fully-grown testis replaced in the abdomen undergoes retrograde changes until it becomes similar in structure. This structure is precisely similar to that found in imperfectly-descended testes. Therefore, from the point of view of procreation, no harrn is at all likely to be done by returning the testis to the abdominal cavity, whilst its subsequent growth and the presence of apparentiy functional cells points to the probable value to the organism of its presence, as for the perfection of the secondary male characters.

Last, I have left the selection of cases which do not need operation. In primis, the extremely difficult task of excluding the co-existence of a hernia must be accomplished. Secondly, in our present state of knowledge it may be taken that the less imperfectly the organ is descended the more likely is it to develop satisfactorily at puberty. That is to say, those cases in which an orchidopexy seems most likely to be successful are the very ones in which the testis may be left alone, for if this operation is to be successful the testis will most probably be close to the scrotum. At the other extreme of the serial degrees of the descent or imperfect descent of the testis, that is, when it is abdominal, it should be left alone.

\section{Summary of Operative Pro edures.}

I. Orchidopexy is only applicable in mild cases of imperfectly-descended testis, and perhap:s even then it may not often be called for.

2. Orchidectomy is only justifiable under special pathological conditions, for example, torsion, severe neuralgia, extreme atrophy and so forth, and in older cases, that is after the occurrence of puberty and a possible and problematical period of testicular activity and spermatogenesis has passed, say from 23 upwards.

3. Replacement in the abdomen is indicated in far the majority of cases, and should be always done before puberty and, perhaps, up to the age of 20 or thereabouts. It would appear that the earlier the operation is performed the better should be the result.

4. No operation may be called for in mild cases when the testis is close to the bottom of the scrotum, or when the testes are abdominally retained.

5. Operative interference is demanded in most cases on account of the secondary changes of an inflammatory and sclerotic nature in the testis, which the position of imperfect descent leads to. Again, there is the frequent coexistence of a hernia with this condition. And in cases where it does not often or has never previously come down, the narrow opening or neck of the sac may cause one of the most dangerous varieties of strangulation.

The Acquired Imperfectly-descended Testis.

As an appendix to the foregoing part of this lecture, it seems advisable to attract attention to the various ways in which an imperfectly-descended condition of the testicle can be acquired. This is almost always the result of an operation for the radical cure of a hernia. Previous to that operation the testis was at the bottom of the scrotum. There are three ways in which this may be brought about. In the first, the testicle was originally imperfectly-descended, but owing to the propulsion of a hernia behind it, the organ has assumed a lower or more descended position. It therefore appears to be fully descended. When at the operation the hernia sac has been freed and divided, ligatured, etc., the testis is apt to resume its original position, so reproducing its imperfect descent. Secondly, if the hernia sac is not sufficiently freed from the cord, and after ligature the sac has been reduced into the abdomen, it will pull the testis up into a higher position. Thirdly, in bandaging up a case after an operation, as for instance the radical cure of a hernia, the organ may become adherent to the scar of the operation. By this is meant the internal rather than the external cicatrix. $I$ do not believe that this last is anything like so frequent as the two former factors in the causation. Similariy the second factor can be excluded in the hands of competent surgeons. And one is led to look upon the first cause, original imperfect descent, as the most important. And also to a a very general conclusion, namely, we recognize that imperfect descent of the testis is frequently accompanied by a hernia; and we may now recognize that a hernia may be accompanied by an imperfectly-descended testis, especially so in children and when the sac is a congenital one. A further generalization may be made. The proper closure of the processus vaginalis that leads to the congenital discontinuity or separation of the tunica vaginalis and the peritoneum is dependent, wholly or in part, on a perfect descent of the testicle and that the failure of this procedure very likely represents one of the earliest and smallest developmental defects, which in a more severe form is recognized as an imperfectly-descended testis. The presence of a congenital sac should always place the surgeon on his guard for the presence of a testicular anomaly. The imperfect descent of the testis is merely an example of a series of degrees of developmental errors.

A great deal of harm may result to the individual from this oversight on the surgeon's part, on account of imperfect maturation of the gland, which becomes exposed to all the changes that result from its position. The condition should be recognized and treated secundum artem at the first operation and it is merely necessary that attention should be called to it for this to be done. Clinical experience emphasizes this point. The consequent healing of the wound with matting of the cord may lead to interference with the blood supply and to impoverishment of the nutrition of the gland. Also the same process does bring about a more or less complete fixation of it, so that later operations for transplanting the organ to the scrotum or the abdomen cannot be carried out, and one is left with the choice of protecting or excising the gland. Recurrent attacks of pain and orchitis usually precipitate the latter procedure. Or the organ wastes and becomes practically a mass of fibrous tissue.

\section{FOUR ABDOMINAL CASHS.}

BY DAVID MACEWAN, M.D., C.M.,

Professor of Surgery, University College, Dundee (University of St. Andrews).

THx following cases which lately came under my care in the Dundee Royal Infirmary are of sufficient interest to make it desirable that they should be recorded:

CASE I.-Hernia into the Ileo.caecal Fossa: Enterectomy.

Mrs. F., aged 56 , admitted December 3 ra, r 1903 .

History.-Illness commenced five days before with abdominal pain and vomiting. The vomiting was incessant for twenty-four hours and then abated somewwhat after a slight movement of the bowels. It only troubled her occasionally for the next three days and then again became very frequent, and continued so till her admission thirty hours became very frequent, and con movement of the bowels or passage of Jater.

State on Admission.-She was very exhausted; pulse weak : temperature $99^{\circ}$. Complained of abdominal pain, chiefly about umbilicus. Abdomen was distended and tympanitic all over. There was pain on palpation, but no localized tenderness. In the left groin at the femoral palpation, but no localinout opening there was a small tumour of doughy consistence and without impulse on coughis.

Operation.-An incision was made down to the tumour in the left groin to determine its nature. It was found to be, not a hernia bu an enlarged gland, and was removed. The abdomen was then opened by a median incision below the umbilicus. The intestines were distended and congested. The obstruction was found to be due to the the mouth of which lay to the inner side of the caecum and appendix. The loop, which was about 4 in. in length, was so constricted that the mouth of the sac had to be incised in order to disengage it. It was distinctly gangrenous, particularly at the constricted parts, which were of an ashy-grey colour, and there was a perforation through which some faeal matter escaped. The sphacelated bowel was then withdrawn from faecal matter escaped. The sphacelated aowel was then withdrawe ends brought together the abdomen, enterectomy performed, and the ends brought together As the pulse was very weak at the end of the operation, 2 pints of saline solution were infused into the median cephalic vein, and a hypodermic injection of strychnine given. The patient made a satisfactory recovery, and the button was passed on January inth, six weeks after the operation.

REMARKs.-I used a Murphy button in this case in preference to suture, on account of the very unequal size of the two openings, due to the dilated state of the bowel above the obstruction; and also because the exhausted state of the patient necessitated as speedy a termination of the operation as possible. Varied descriptions have been given of the peritoneal fossae in the region of the caecum. The three principal fossae are the ileo-colic, the ileo-caecal or ileoappendicular, and the subcaecal or retro-colic. They are rarely the seats of hernia, and in the cases which have been recorded the condition has usually been discovered on postmartem examination. Hernia into the ileo-colic fossa has not been observed. Moynihan in. his work on retroperitoneal 